\title{
Blow-up phenomena and global existence for the periodic two-component Dullin-Gottwald-Holm system
}

Jingjing Liu ${ }^{1 *}$ and Dequan Zhang ${ }^{2}$

"Correspondence:

jingjing830306@163.com

1 Department of Mathematics and

Information Science, Zhengzhou

University of Light Industry,

Zhengzhou, 450002, China

Full list of author information is

available at the end of the article

\begin{abstract}
This paper is concerned with blow-up phenomena and global existence for the periodic two-component Dullin-Gottwald-Holm system. We first obtain several blow-up results and the blow-up rate of strong solutions to the system. We then present a global existence result for strong solutions to the system.
\end{abstract}

MSC: $35 \mathrm{G} 25 ; 35 \mathrm{~L} 05$

Keywords: periodic two-component Dullin-Gottwald-Holm system; blow-up; blow-up rate; global existence

\section{Introduction}

In this paper, we consider the following periodic two-component Dullin-Gottwald-Holm (DGH) system:

$$
\left\{\begin{array}{l}
m_{t}-A u_{x}+u m_{x}+2 u_{x} m+\gamma u_{x x x}+\rho \rho_{x}=0, \quad t>0, x \in \mathbb{R} \\
\rho_{t}+(u \rho)_{x}=0, \quad t>0, x \in \mathbb{R} \\
u(0, x)=u_{0}(x), \quad x \in \mathbb{R} \\
\rho(0, x)=\rho_{0}(x), \quad x \in \mathbb{R} \\
u(t, x+1)=u(t, x), \quad t \geq 0, x \in \mathbb{R} \\
\rho(t, x+1)=\rho(t, x), \quad t \geq 0, x \in \mathbb{R}
\end{array}\right.
$$

where $m=u-u_{x x}, A>0$ and $\gamma$ are constants.

System (1.1) has been recently derived by Zhu et al. in [1] by following Ivanov's approach [2]. It was shown in [1] that the DGH system is completely integrable and can be written as a compatibility condition of two linear systems

$$
\Psi_{x x}=\left(-\xi^{2} \rho^{2}+\xi\left(m-\frac{A}{2}+\frac{\gamma}{2}\right)+\frac{1}{4}\right) \Psi
$$

and

$$
\Psi_{t}=\left(\frac{1}{2 \xi}-u+\gamma\right) \Psi_{x}+\frac{1}{2} u_{x} \Psi,
$$

๑ 2013 Liu and Zhang; licensee Springer. This is an Open Access article distributed under the terms of the Creative Commons Attribution License (http://creativecommons.org/licenses/by/2.0), which permits unrestricted use, distribution, and reproduction in any medium, provided the original work is properly cited. 
where $\xi$ is a spectral parameter. Moreover, this system has the following two Hamiltonians:

$$
E(u, \rho)=\frac{1}{2} \int\left(u^{2}+u_{x}^{2}+(\rho-1)^{2}\right) d x
$$

and

$$
F(u, \rho)=\frac{1}{2} \int\left(u^{3}+u u_{x}^{2}-A u^{2}-\gamma u_{x}^{2}+2 u(\rho-1)+u(\rho-1)^{2}\right) d x .
$$

For $\rho=0$ and $m=u-\alpha^{2} u_{x x}$, (1.1) becomes the DGH equation [3]

$$
u_{t}-\alpha^{2} u_{t x x}-A u_{x}+3 u u_{x}+\gamma u_{x x x}=\alpha^{2}\left(2 u_{x} u_{x x}+u u_{x x x}\right)
$$

where $A$ and $\alpha$ are two positive constants, modeling unidirectional propagation of surface waves on a shallow layer of water which is at rest at infinity, $u(t, x)$ stands for fluid velocity. It is completely integrable with a bi-Hamiltonian and a Lax pair. Moreover, its traveling wave solutions include both the $\mathrm{KdV}$ solitons and the $\mathrm{CH}$ peakons as limiting cases [3]. The Cauchy problem of the DGH equation has been extensively studied, $c f$. [4-13].

For $\rho \neq \equiv, \gamma=0$, system (1.1) becomes the two-component Camassa-Holm system [2]

$$
\left\{\begin{array}{l}
m_{t}-A u_{x}+u m_{x}+2 u_{x} m+\rho \rho_{x}=0 \\
\rho_{t}+(u \rho)_{x}=0
\end{array}\right.
$$

where $\rho(t, x)$ is in connection with the free surface elevation from scalar density (or equilibrium), and the parameter $A$ characterizes a linear underlying shear flow. System (1.2) describes water waves in the shallow water regime with nonzero constant vorticity, where the nonzero vorticity case indicates the presence of an underlying current. A large amount of literature was devoted to the Cauchy problem (1.2); see [14-22].

The Cauchy problem (1.1) has been discussed in [1]. Therein Zhu and Xu established the local well-posedness to system (1.1), derived the precise blow-up scenario and investigated the wave breaking for it. The aim of this paper is to further study the blow-up phenomena for strong solutions to (1.1) and to present a global existence result.

Our paper is organized as follows. In Section 2, we briefly state some needed results including the local well posedness of system (1.1), the precise blow-up scenario and some useful lemmas to study blow-up phenomena and global existence. In Section 3, we give several new blow-up results and the precise blow-up rate. In Section 4, we present a new global existence result of strong solutions to (1.1).

Notation Given a Banach space $Z$, we denote its norm by $\|\cdot\|_{Z}$. Since all space of functions is over $\mathbb{S}$, for simplicity, we drop $\mathbb{S}$ in our notations if there is no ambiguity.

\section{Preliminaries}

In this section, we will briefly give some needed results in order to pursue our goal. 
With $m=u-u_{x x}$, we can rewrite system (1.1) as follows:

$$
\left\{\begin{array}{l}
u_{t}-u_{t x x}-A u_{x}+\gamma u_{x x x}+3 u u_{x}-2 u_{x} u_{x x}-u u_{x x x}+\rho \rho_{x}=0, \quad t>0, x \in \mathbb{R}, \\
\rho_{t}+(u \rho)_{x}=0, \quad t>0, x \in \mathbb{R}, \\
u(0, x)=u_{0}(x), \quad x \in \mathbb{R}, \\
\rho(0, x)=\rho_{0}(x), \quad x \in \mathbb{R}, \\
u(t, x+1)=u(t, x), \quad t \geq 0, x \in \mathbb{R} \\
\rho(t, x+1)=\rho(t, x), \quad t \geq 0, x \in \mathbb{R} .
\end{array}\right.
$$

Note that if $G(x):=\frac{\cosh (x-[x]-1 / 2)}{2 \sinh (1 / 2)}, x \in \mathbb{R}$ is the kernel of $\left(1-\partial_{x}^{2}\right)^{-1}$, then $\left(1-\partial_{x}^{2}\right)^{-1} f=G * f$ for all $f \in L^{2}(\mathbb{S}), G * m=u$. Here we denote by $*$ the convolution. Using this identity, we can rewrite system (2.1) as follows:

$$
\left\{\begin{array}{l}
u_{t}+(u-\gamma) u_{x}=-\partial_{x} G *\left(u^{2}+\frac{1}{2} u_{x}^{2}+(\gamma-A) u+\frac{1}{2} \rho^{2}\right), \quad t>0, x \in \mathbb{R}, \\
\rho_{t}+(u \rho)_{x}=0, \quad t>0, x \in \mathbb{R}, \\
u(0, x)=u_{0}(x), \quad x \in \mathbb{R}, \\
\rho(0, x)=\rho_{0}(x), \quad x \in \mathbb{R}, \\
u(t, x+1)=u(t, x), \quad t \geq 0, x \in \mathbb{R}, \\
\rho(t, x+1)=\rho(t, x), \quad t \geq 0, x \in \mathbb{R} .
\end{array}\right.
$$

The local well-posedness of the Cauchy problem (2.1) can be obtained by applying Kato's theorem. As a result, we have the following well-posedness result.

Lemma 2.1 [1] Given the initial data $\left(u_{0}, \rho_{0}\right) \in H^{s} \times H^{s-1}, s \geq 2$, there exists a maximal $T=T\left(\left\|\left(u_{0}, \rho_{0}\right)\right\|_{H^{s} \times H^{s-1}}\right)>0$ and a unique solution

$$
(u, \rho) \in C\left([0, T) ; H^{s} \times H^{s-1}\right) \cap C^{1}\left([0, T) ; H^{s-1} \times H^{s-2}\right)
$$

of (2.1). Moreover, the solution $(u, \rho)$ depends continuously on the initial data $\left(u_{0}, \rho_{0}\right)$ and the maximal time of existence $T>0$ is independent of s.

Consider now the following initial value problem:

$$
\begin{cases}q_{t}=u(t, q), & t \in[0, T) \\ q(0, x)=x, & x \in \mathbb{R}\end{cases}
$$

where $u$ denotes the first component of the solution $(u, \rho)$ to (2.1).

Lemma 2.2 [1] Let $(u, \rho)$ be the solution of $(2.1)$ with the initial data $\left(u_{0}, \rho_{0}\right) \in H^{s} \times H^{s-1}$, $s \geq 2$. Then Eq. (2.3) has a unique solution $q \in C^{1}([0, T) \times \mathbb{R} ; \mathbb{R})$. Moreover, the map $q(t, \cdot)$ is an increasing diffeomorphism of $\mathbb{R}$ with

$$
q_{x}(t, x)=\exp \left(\int_{0}^{t} u_{x}(s, q(s, x)) d s\right)>0, \quad(t, x) \in[0, T) \times \mathbb{R} .
$$


Lemma $2.3[1]$ Let $(u, \rho)$ be the solution of $(2.1)$ with the initial data $\left(u_{0}, \rho_{0}\right) \in H^{s} \times H^{s-1}$, $s \geq 2$, and $T>0$ be the maximal time of existence. Then we have

$$
\rho(t, q(t, x)) q_{x}(t, x)=\rho_{0}(x), \quad(t, x) \in[0, T) \times \mathbb{S} .
$$

Moreover, if there exists an $x_{0} \in \mathbb{S}$ such that $\rho_{0}\left(x_{0}\right)=0$, then $\rho\left(t, q\left(t, x_{0}\right)\right)=0$ for all $t \in$ $[0, T)$.

Next, we give two useful conservation laws of strong solutions to (2.1).

Lemma 2.4 [1] Let $(u, \rho)$ be the solution of $(2.1)$ with the initial data $\left(u_{0}, \rho_{0}\right) \in H^{s} \times H^{s-1}$, $s \geq 2$, and let $T>0$ be the maximal time of existence. Then, for all $t \in[0, T)$, we have

$$
\int_{\mathbb{S}}\left(u^{2}+u_{x}^{2}+\rho^{2}\right) d x=\int_{\mathbb{S}}\left(u_{0}^{2}+u_{0, x}^{2}+\rho_{0}^{2}\right) d x:=E_{0} .
$$

Lemma 2.5 Let $(u, \rho)$ be the solution of (2.1) with the initial data $\left(u_{0}, \rho_{0}\right) \in H^{s} \times H^{s-1}$, $s \geq 2$, and let $T>0$ be the maximal time of existence. Then, for all $t \in[0, T)$, we have

$$
\int_{\mathbb{S}} u(t, x) d x=\int_{\mathbb{S}} u_{0}(x) d x
$$

Proof By the first equation in (2.1), we have

$$
\begin{aligned}
\frac{d}{d t} \int_{\mathbb{S}} u(t, x) d x & =\int_{\mathbb{S}} u_{t} d x \\
& =\int_{\mathbb{S}}\left(u_{t x x}+A u_{x}-\gamma u_{x x x}-3 u u_{x}+2 u_{x} u_{x x}+u u_{x x x}-\rho \rho_{x}\right) d x=0
\end{aligned}
$$

This completes the proof of the lemma.

Then we state the following precise blow-up mechanism of (2.1).

Lemma 2.6 [1] Let $(u, \rho)$ be the solution of $(2.1)$ with the initial data $\left(u_{0}, \rho_{0}\right) \in H^{s} \times H^{s-1}$, $s \geq 2$, and let $T>0$ be the maximal time of existence. Then the solution blows up in finite time if and only if

$$
\liminf _{t \rightarrow T^{-}}\left\{\inf _{x \in \mathbb{S}} u_{x}(t, x)\right\}=-\infty
$$

Lemma $2.7[23]$ Let $t_{0}>0$ and $v \in C^{1}\left(\left[0, t_{0}\right) ; H^{2}(\mathbb{R})\right)$. Then, for every $t \in\left[0, t_{0}\right)$, there exists at least one point $\xi(t) \in \mathbb{R}$ with

$$
m(t):=\inf _{x \in \mathbb{R}}\left\{v_{x}(t, x)\right\}=v_{x}(t, \xi(t))
$$

and the function $m$ is almost everywhere differentiable on $\left(0, t_{0}\right)$ with

$$
\frac{d}{d t} m(t)=v_{t x}(t, \xi(t)) \quad \text { a.e. on }\left(0, t_{0}\right)
$$


Lemma 2.8 [24] (i) For every $f \in H^{1}(\mathbb{S})$, we have

$$
\max _{x \in[0,1]} f^{2}(x) \leq \frac{e+1}{2(e-1)}\|f\|_{H^{1}}^{2}
$$

where the constant $\frac{e+1}{2(e-1)}$ is sharp.

(ii) For every $f \in H^{3}(\mathbb{S})$, we have

$$
\max _{x \in[0,1]} f^{2}(x) \leq c\|f\|_{H^{1}}^{2}
$$

with the best possible constant c lying within the range $\left(1, \frac{13}{12}\right]$. Moreover, the best constant $c$ is $\frac{e+1}{2(e-1)}$.

Lemma 2.9 [25] If $\in H^{3}(\mathbb{S})$ is such that $\int_{\mathbb{S}} f(x) d x=\frac{a_{0}}{2}$, then, for every $\epsilon>0$, we have

$$
\max _{x \in[0,1]} f^{2}(x) \leq \frac{\epsilon+2}{24} \int_{\mathbb{S}} f_{x}^{2} d x+\frac{\epsilon+2}{4 \epsilon} a_{0}^{2} .
$$

Moreover,

$$
\max _{x \in[0,1]} f^{2}(x) \leq \frac{\epsilon+2}{24}\|f\|_{H^{1}(\mathbb{S})}^{2}+\frac{\epsilon+2}{4 \epsilon} a_{0}^{2} .
$$

Lemma 2.10 [26] Assume that a differentiable function $y(t)$ satisfies

$$
y^{\prime}(t) \leq-C y^{2}(t)+K
$$

where $C, K$ are positive constants. If the initial datum $y(0)=y_{0}<-\sqrt{\frac{K}{C}}$, then the solution to (2.4) goes to $-\infty$ before $t$ tends to $\frac{1}{-C y_{0}+\frac{K}{y_{0}}}$.

\section{Blow-up phenomena}

In this section, we discuss the blow-up phenomena of system (2.1). Firstly, we prove that there exist strong solutions to (2.1) which do not exist globally in time.

Theorem 3.1 Let $\left(u_{0}, \rho_{0}\right) \in H^{s} \times H^{s-1}, s \geq 2$, and $T$ be the maximal time of the solution $(u, \rho)$ to $(2.1)$ with the initial data $\left(u_{0}, \rho_{0}\right)$. If there is some $x_{0} \in \mathbb{S}$ such that $\rho_{0}\left(x_{0}\right)=0$ and

$$
u_{0}^{\prime}\left(x_{0}\right)=\inf _{x \in \mathbb{S}} u_{0}^{\prime}(x)<-\sqrt{\frac{e+1}{2(e-1)} E_{0}+|\gamma-A| \sqrt{\frac{8(e+1)}{e-1}} E_{0}^{\frac{1}{2}}},
$$

then the corresponding solution to (2.1) blows up in finite time.

Proof Applying Lemma 2.1 and a simple density argument, we only need to show that the above theorem holds for some $s \geq 2$. Here we assume $s=3$ to prove the above theorem.

Define now

$$
m(t):=\inf _{x \in \mathbb{S}}\left[u_{x}(t, x)\right], \quad t \in[0, T) .
$$


By Lemma 2.7, we let $\xi(t) \in \mathbb{S}$ be a point where this infimum is attained. It follows that

$$
m(t)=u_{x}(t, \xi(t)) \quad \text { and } \quad u_{x x}(t, \xi(t))=0
$$

Differentiating the first equation in (2.2) with respect to $x$ and using the identity $\partial_{x}^{2} G * f=$ $G * f-f$, we have

$$
\begin{aligned}
u_{t x}+(u-\gamma) u_{x x}= & -\frac{1}{2} u_{x}^{2}+\frac{1}{2} \rho^{2}+u^{2}+(\gamma-A) u \\
& -G *\left(u^{2}+\frac{1}{2} u_{x}^{2}+\frac{1}{2} \rho^{2}+(\gamma-A) u\right) .
\end{aligned}
$$

Since the map $q(t, \cdot)$ given by (2.3) is an increasing diffeomorphism of $\mathbb{R}$, there exists a $x(t) \in \mathbb{S}$ such that $q(t, x(t))=\xi(t)$. In particular, $x(0)=\xi(0)$. Note that $u_{0}^{\prime}\left(x_{0}\right)=\inf _{x \in \mathbb{S}} u_{0}^{\prime}(x)$, we can choose $x_{0}=\xi(0)$. It follows that $x(0)=\xi(0)=x_{0}$. By Lemma 2.3 and the condition $\rho_{0}\left(x_{0}\right)=0$, we have

$$
\rho(t, \xi(t)) q_{x}(t, x)=\rho(t, q(t, x(t))) q_{x}(t, x)=\rho_{0}(x(0))=\rho_{0}\left(x_{0}\right)=0 .
$$

Thus $\rho(t, \xi(t))=0$.

Valuating (3.1) at $(t, \xi(t))$ and using Lemma 2.7, we obtain

$$
\frac{d m(t)}{d t} \leq-\frac{1}{2} m^{2}(t)+\frac{1}{2} u^{2}+(\gamma-A) u-(\gamma-A) G * u,
$$

here we used the relations $G *\left(u^{2}+\frac{1}{2} u_{x}^{2}\right) \geq \frac{1}{2} u^{2}$ and $G * \rho^{2} \geq 0$. Note that $\|G\|_{L^{1}}=1$. By Lemma 2.4 and Lemma 2.8 , we get

$$
\begin{aligned}
& \|u\|_{L^{\infty}}^{2} \leq \frac{e+1}{2(e-1)}\|u\|_{H^{1}}^{2} \leq \frac{e+1}{2(e-1)} E_{0}, \\
& |(\gamma-A) u| \leq|\gamma-A|\|u\|_{L^{\infty}} \leq|\gamma-A| \sqrt{\frac{e+1}{2(e-1)}} E_{0}^{\frac{1}{2}}
\end{aligned}
$$

and

$$
|(\gamma-A) G * u| \leq|\gamma-A|\|G\|_{L^{1}}\|u\|_{L^{\infty}} \leq|\gamma-A| \sqrt{\frac{e+1}{2(e-1)}} E_{0}^{\frac{1}{2}} .
$$

It follows that

$$
\frac{d m(t)}{d t} \leq-\frac{1}{2} m^{2}(t)+K
$$

where $K=\frac{e+1}{4(e-1)} E_{0}+2|\gamma-A| \sqrt{\frac{e+1}{2(e-1)}} E_{0}^{\frac{1}{2}}$. Since $m(0)<-\sqrt{2 K}$, Lemma 2.10 implies

$$
\lim _{t \rightarrow T} m(t)=-\infty \quad \text { with } T=\frac{2 u_{0}^{\prime}\left(x_{0}\right)}{2 K-\left(u_{0}^{\prime}\left(x_{0}\right)\right)^{2}}
$$

Applying Lemma 2.6, the solution blows up in finite time. 
Theorem 3.2 Let $\left(u_{0}, \rho_{0}\right) \in H^{s} \times H^{s-1}, s \geq 2$, and $T$ be the maximal time of the solution $(u, \rho)$ to (2.1) with the initial data $\left(u_{0}, \rho_{0}\right)$. Assume that $\int_{\mathbb{S}} u_{0}(x) d x=\frac{a_{0}}{2}$. If there is some $x_{0} \in \mathbb{S}$ such that $\rho_{0}\left(x_{0}\right)=0$ and for any $\epsilon>0$,

$$
u_{0}^{\prime}\left(x_{0}\right)=\inf _{x \in \mathbb{S}} u_{0}^{\prime}(x)<-\sqrt{\frac{\epsilon+2}{24} E_{0}+\frac{\epsilon+2}{4 \epsilon} a_{0}^{2}+|\gamma-A| \sqrt{\frac{2(\epsilon+2)}{3} E_{0}+\frac{4(\epsilon+2)}{\epsilon} a_{0}^{2}}},
$$

then the corresponding solution to (2.1) blows up in finite time.

Proof By Lemma 2.5, we have $\int_{\mathbb{S}} u(t, x) d x=\frac{a_{0}}{2}$. Using Lemma 2.4 and Lemma 2.9, we obtain

$$
\begin{aligned}
& \|u\|_{L^{\infty}}^{2} \leq \frac{\epsilon+2}{24} E_{0}+\frac{\epsilon+2}{4 \epsilon} a_{0}^{2}, \\
& |(\gamma-A) u| \leq|\gamma-A|\|u\|_{L^{\infty}} \leq|\gamma-A| \sqrt{\frac{\epsilon+2}{24} E_{0}+\frac{\epsilon+2}{4 \epsilon} a_{0}^{2}}
\end{aligned}
$$

and

$$
|(\gamma-A) G * u| \leq|\gamma-A|\|G\|_{L^{1}}\|u\|_{L^{\infty}} \leq|\gamma-A| \sqrt{\frac{\epsilon+2}{24} E_{0}+\frac{\epsilon+2}{4 \epsilon} a_{0}^{2}} .
$$

Following a similar proof in Theorem 3.1, we have

$$
\frac{d m(t)}{d t} \leq-\frac{1}{2} m^{2}(t)+K
$$

where $K=\frac{\epsilon+2}{48} E_{0}+\frac{\epsilon+2}{8 \epsilon} a_{0}^{2}+|\gamma-A| \sqrt{\frac{\epsilon+2}{6} E_{0}+\frac{\epsilon+2}{\epsilon} a_{0}^{2}}$. Following the same argument as in Theorem 3.1, we deduce that the solution blows up in finite time.

Letting $a_{0}=0$ and $\epsilon \rightarrow 0$ in Theorem 3.2, we have the following result.

Corollary 3.1 Let $\left(u_{0}, \rho_{0}\right) \in H^{s} \times H^{s-1}, s \geq 2$, and $T$ be the maximal time of the solution $(u, \rho)$ to (2.1) with the initial data $\left(u_{0}, \rho_{0}\right)$. Assume that $\int_{\mathbb{S}} u_{0}(x) d x=0$. If there is some $x_{0} \in \mathbb{S}$ such that $\rho_{0}\left(x_{0}\right)=0$ and

$$
u_{0}^{\prime}\left(x_{0}\right)=\inf _{x \in \mathbb{S}} u_{0}^{\prime}(x)<-\sqrt{\frac{E_{0}}{12}+2|\gamma-A| \sqrt{\frac{E_{0}}{3}}}
$$

then the corresponding solution to (2.1) blows up in finite time.

Remark 3.1 Note that system (2.1) is variational under the transformation $(u, x) \rightarrow$ $(-u,-x)$ and $(\rho, x) \rightarrow(\rho,-x)$ even $\gamma=0$. Thus, we cannot get a blow-up result according to the parity of the initial data $\left(u_{0}, \rho_{0}\right)$ as we usually do.

Next, we will give more insight into the blow-up mechanism for the wave-breaking solution to system (2.1), that is, the blow-up rate for strong solutions to (2.1). 
Theorem 3.3 Let $(u, \rho)$ be the solution to system (2.1) with the initial data $\left(u_{0}, \rho_{0}\right) \in H^{s} \times$ $H^{s-1}, s \geq 2$, satisfying the assumption of Theorem 3.1, and $T$ be the maximal time of the solution $(u, \rho)$. Then we have

$$
\lim _{t \rightarrow T}(T-t) \inf _{x \in \mathbb{S}} u_{x}(t, x)=-2
$$

Proof As mentioned earlier, here we only need to show that the above theorem holds for $s=3$.

Define now

$$
m(t):=\inf _{x \in \mathbb{S}}\left[u_{x}(t, x)\right], \quad t \in[0, T)
$$

By the proof of Theorem 3.1, there exists a positive constant $K=K\left(E_{0}, \gamma, A\right)$ such that

$$
-K \leq \frac{d}{d t} m+\frac{1}{2} m^{2} \leq K \quad \text { a.e. on }(0, T) .
$$

Let $\varepsilon \in\left(0, \frac{1}{2}\right)$. Since $\liminf _{t \rightarrow T} m(t)=-\infty$ by Theorem 3.1 , there is some $t_{0} \in(0, T)$ with $m\left(t_{0}\right)<0$ and $m^{2}\left(t_{0}\right)>\frac{K}{\varepsilon}$. Since $m$ is locally Lipschitz, it is then inferred from (3.5) that

$$
m^{2}(t)>\frac{K}{\varepsilon}, \quad t \in\left[t_{0}, T\right)
$$

A combination of (3.5) and (3.6) enables us to infer

$$
\frac{1}{2}+\varepsilon \geq-\frac{\frac{d m}{d t}}{m^{2}} \geq \frac{1}{2}-\varepsilon \quad \text { a.e. on }(0, T)
$$

Since $m$ is locally Lipschitz on $[0, T)$ and (3.6) holds, it is easy to check that $\frac{1}{m}$ is locally Lipschitz on $\left(t_{0}, T\right)$. Differentiating the relation $m(t) \cdot \frac{1}{m(t)}=1, t \in\left(t_{0}, T\right)$, we get

$$
\frac{d}{d t}\left(\frac{1}{m}\right)=-\frac{\frac{d m}{d t}}{m^{2}} \quad \text { a.e. on }\left(t_{0}, T\right)
$$

with $\frac{1}{m}$ absolutely continuous on $\left(t_{0}, T\right)$. For $t \in\left(t_{0}, T\right)$. Integrating (3.7) on $(t, T)$ to obtain

$$
\left(\frac{1}{2}+\varepsilon\right)(T-t) \geq-\frac{1}{m(t)} \geq\left(\frac{1}{2}-\varepsilon\right)(T-t), \quad t \in\left(t_{0}, T\right)
$$

that is

$$
\frac{1}{\frac{1}{2}+\varepsilon} \leq-m(t)(T-t) \leq \frac{1}{\frac{1}{2}-\varepsilon}, \quad t \in\left(t_{0}, T\right) .
$$

By the arbitrariness of $\varepsilon \in\left(0, \frac{1}{2}\right)$ the statement of Theorem 3.3 follows.

\section{Global existence}

In this section, we will present a global existence result. 
Theorem 4.1 Let $\left(u_{0}, \rho_{0}\right) \in H^{s} \times H^{s-1}, s \geq 2$, and $T$ be the maximal time of the solution $(u, \rho)$ to $(2.1)$ with the initial data $\left(u_{0}, \rho_{0}\right)$. If $\rho_{0}(x) \neq 0$ for all $x \in \mathbb{S}$, then the corresponding solution $(u, \rho)$ exists globally in time.

Proof Define

$$
m(t):=\inf _{x \in \mathbb{S}}\left[u_{x}(t, x)\right], \quad t \in[0, T)
$$

By Lemma 2.7, we let $\xi(t) \in \mathbb{S}$ be a point where this infimum is attained. It follows that

$$
m(t)=u_{x}(t, \xi(t)) \quad \text { and } \quad u_{x x}(t, \xi(t))=0 .
$$

Since the map $q(t, \cdot)$ given by (2.3) is an increasing diffeomorphism of $\mathbb{R}$, there exists an $x(t) \in \mathbb{S}$ such that $q(t, x(t))=\xi(t)$.

Set $m(t)=u_{x}(t, \xi(t))=u_{x}(t, q(t, x(t)))$ and $\alpha(t)=\rho(t, \xi(t))=\rho(t, q(t, x(t)))$. Valuating (3.1) at $(t, \xi(t))$ and using Lemma 2.7, we obtain

$$
m^{\prime}(t)=-\frac{1}{2} m^{2}(t)+\frac{1}{2} \alpha^{2}(t)+f \quad \text { and } \quad \alpha^{\prime}(t)=-m(t) \alpha(t),
$$

where $f=u^{2}+(\gamma-A) u-G *\left(u^{2}+\frac{1}{2} u_{x}^{2}+\frac{1}{2} \rho^{2}+(\gamma-A) u\right)$. By Lemma 2.4, Lemma 2.8 and $\frac{1}{2 \sinh \frac{1}{2}} \leq G(x) \leq \frac{\cosh \frac{1}{2}}{2 \sinh \frac{1}{2}}$, we have

$$
\begin{aligned}
|f| & \leq\|u\|_{L^{\infty}}^{2}+2|\gamma-A|\|u\|_{L^{\infty}}+\|G\|_{L^{\infty}}\left\|u^{2}+\frac{1}{2} u_{x}^{2}+\frac{1}{2} \rho^{2}\right\|_{L^{1}} \\
& \leq \frac{e+1}{2(e-1)} E_{0}+2|\gamma-A| \sqrt{\frac{e+1}{2(e-1)}} E_{0}^{\frac{1}{2}}+\frac{\cosh \frac{1}{2}}{2 \sinh \frac{1}{2}} E_{0}:=c_{1} .
\end{aligned}
$$

By Lemmas 2.2-2.3, we know that $\alpha(t)$ has the same sign with $\alpha(0)=\rho_{0}\left(x_{0}\right)$ for every $x \in \mathbb{R}$. Moreover, there is a constant $\beta>0$ such that $|\alpha(0)|=\inf _{x \in \mathbb{S}}\left|\rho_{0}(x)\right| \geq \beta>0$ because of $\rho_{0}(x) \neq 0$ for all $x \in \mathbb{S}$. Next, we consider the following Lyapunov positive function:

$$
w(t)=\alpha(0) \alpha(t)+\frac{\alpha(0)}{\alpha(t)}\left(1+m^{2}(t)\right), \quad t \in[0, T) .
$$

Letting $t=0$ in (4.2), we have

$$
w(0) \leq\left\|\rho_{0}\right\|_{L^{\infty}}^{2}+1+\left\|u_{0}^{\prime}(x)\right\|_{L^{\infty}}^{2}:=c_{2} .
$$

Differentiating (4.2) with respect to $t$ and using (4.1), we obtain

$$
\begin{aligned}
w^{\prime}(t) & =\frac{\alpha(0)}{\alpha(t)} \cdot 2 m(t)\left(f+\frac{1}{2}\right) \\
& \leq \frac{\alpha(0)}{\alpha(t)}\left(1+m^{2}(t)\right)\left(|f|+\frac{1}{2}\right) \\
& \leq w(t)\left(c_{1}+\frac{1}{2}\right) .
\end{aligned}
$$


By Gronwall's inequality, we have

$$
w(t) \leq w(0) e^{\left(c_{1}+\frac{1}{2}\right) t} \leq c_{2} e^{\left(c_{1}+\frac{1}{2}\right) t}
$$

for all $t \in[0, T)$. On the other hand,

$$
w(t) \geq 2 \sqrt{\alpha^{2}(0)\left(1+m^{2}(t)\right)} \geq 2 \beta|m(t)|, \quad \forall t \in[0, T) .
$$

Thus,

$$
|m(t)| \leq \frac{1}{2 \beta} w(t) \leq \frac{c_{2}}{2 \beta} e^{\left(c_{1}+\frac{1}{2}\right) t}
$$

for all $t \in[0, T)$. It follows that

$$
\liminf _{t \rightarrow T} m(t) \geq-\frac{c_{2}}{2 \beta} e^{\left(c_{1}+\frac{1}{2}\right) T} .
$$

This completes the proof by using Lemma 2.6 .

\section{Competing interests}

The authors declare that they have no competing interests.

\section{Authors' contributions}

This paper is the result of joint work of all authors who contributed equally to the final version of this paper. All authors read and approved the final manuscript.

\section{Author details}

${ }^{1}$ Department of Mathematics and Information Science, Zhengzhou University of Light Industry, Zhengzhou, 450002 ,

China. ${ }^{2}$ Faculty of Science, Guilin University of Aerospace Industry, Guilin, 541004, China.

\section{Acknowledgements}

The authors would like to thank the editors and the referees for their valuable suggestions to improve the quality of this paper. This research is partially supported by the Doctoral Research Foundation of Zhengzhou University of Light Industry.

\section{Received: 24 April 2013 Accepted: 16 June 2013 Published: 1 July 2013}

\section{References}

1. Zhu, $\mathrm{M}, \mathrm{Xu}$, J: On the wave-breaking phenomena for the periodic two-component Dullin-Gottwald-Holm system. J. Math. Anal. Appl. 391, 415-428 (2012)

2. Ivanov, R: Two-component integrable systems modelling shallow water waves: the constant vorticity case. Wave Motion 46, 389-396 (2009)

3. Dullin, HR, Gottwald, GA, Holm, DD: An integral shallow water equation with linear and nonlinear dispersion. Phys. Rev. Lett. 87, 4501-4504 (2001)

4. Ai, X, Gui, G: On the inverse scattering problem and the low regularity solutions for the Dullin-Gottwald-Holm equation. Nonlinear Anal., Real World Appl. 11, 888-894 (2010)

5. Liu, Y: Global existence and blow-up solutions for a nonlinear shallow water equation. Math. Ann. 335, 717-735 (2006)

6. Meng, $\mathrm{Q}, \mathrm{He}, \mathrm{B}, \mathrm{L}$ Long, $\mathrm{Y}, \mathrm{Li}$, Z: New exact periodic wave solutions for the Dullin-Gottwald-Holm equation. Appl. Math. Comput. 218, 4533-4537 (2011)

7. Mustafa, OG: Global conservative solutions of the Dullin-Gottwald-Holm equation. Discrete Contin. Dyn. Syst. 19, 575-594 (2007)

8. Tian, L, Gui, G, Liu, Y: On the Cauchy problem and the scattering problem for the Dullin-Gottwald-Holm equation. Commun. Math. Phys. 257, 667-701 (2005)

9. Yan, K, Yin, Z: On the solutions of the Dullin-Gottwald-Holm equation in Besov spaces. Nonlinear Anal., Real World Appl. 13, 2580-2592 (2012)

10. Yin, Z: Well-posedness, blowup, and global existence for an integrable shallow water equation. Discrete Contin. Dyn. Syst. 11, 393-411 (2004)

11. Yin, Z: Global existence and blow-up for a periodic integrable shallow water equation with linear and nonlinear dispersion. Dyn. Contin. Discrete Impuls. Syst., Ser. A Math. Anal. 12, 87-101 (2005)

12. Zhang, S, Yin, Z: On the blow-up phenomena of the periodic Dullin-Gottwald-Holm equation. J. Math. Phys. 49, 1-16 (2008) 
13. Zhang, S, Yin, Z: Global weak solutions for the Dullin-Gottwald-Holm equation. Nonlinear Anal. 72, 1690-1700 (2010)

14. Chen, RM, Liu, Y: Wave-breaking and global existence for a generalized two-component Camassa-Holm system. Int Math. Res. Not. 2011, 1381-1416 (2011)

15. Chen, RM, Liu, Y, Qiao, Z: Stability of solitary waves of a generalized two-component Camassa-Holm system. Commun. Partial Differ. Equ. 36, 2162-2188 (2011)

16. Constantin, A, Ivanov, R: On the integrable two-component Camassa-Holm shallow water system. Phys. Lett. A 372, 7129-7132 (2008)

17. Escher, J, Lechtenfeld, O, Yin, Z: Well-posedness and blow-up phenomena for the 2-component Camassa-Holm equation. Discrete Contin. Dyn. Syst. 19, 493-513 (2007)

18. Fu, Y, Liu, Y, Qu, C: Well-posedness and blow-up solution for a modified two-component periodic Camassa-Holm system with peakons. Math. Ann. 348, 415-448 (2010)

19. Gui, G, Liu, Y: On the Cauchy problem for the two-component Camassa-Holm system. Math. Z. 268, 45-66 (2010)

20. Gui, G, Liu, Y: On the global existence and wave-breaking criteria for the two-component Camassa-Holm system. J. Funct. Anal. 258, 4251-4278 (2010)

21. Hu, Q: On a periodic 2-component Camassa-Holm equation with vorticity. J. Nonlinear Math. Phys. 18, 541-556 (2011)

22. Zhang, P, Liu, Y: Stability of solitary waves and wave-breaking phenomena for the two-component Camassa-Holm system. Int. Math. Res. Not. 11, 1981-2021 (2010)

23. Constantin, A, Escher, J: Wave breaking for nonlinear nonlocal shallow water equations. Acta Math. 181, 229-243 (1998)

24. Yin, Z: On the blow-up of solutions of the periodic Camassa-Holm equation. Dyn. Contin. Discrete Impuls. Syst., Ser. A Math. Anal. 12,375-381 (2005)

25. Hu, Q, Yin, Z: Blowup phenomena for a new periodic nonlinearly dispersive wave equation. Math. Nachr. 283(11) 1613-1628 (2010)

26. Zhou, Y: Blow-up of solutions to a nonlinear dispersive rod equation. Calc. Var. Partial Differ. Equ. 25, 63-77 (2005)

doi:10.1186/1687-2770-2013-158

Cite this article as: Liu and Zhang: Blow-up phenomena and global existence for the periodic two-component Dullin-Gottwald-Holm system. Boundary Value Problems 2013 2013:158.

\section{Submit your manuscript to a SpringerOpen ${ }^{\odot}$ journal and benefit from:}

- Convenient online submission

- Rigorous peer review

- Immediate publication on acceptance

- Open access: articles freely available online

- High visibility within the field

- Retaining the copyright to your article 